\title{
The effect of an overall healthy lifestyle on early-onset stroke: a cross-sectional study
}

\author{
Cui Wang $^{1 \#}$, Xiao-Lin Huang ${ }^{1 \#}$, Shou-Kui Xiang ${ }^{1}$, Xue-Gan Lian ${ }^{2}$, Cheng Yuan ${ }^{1}$, Xiao-Hong Jiang ${ }^{1}$, \\ Fei Hua ${ }^{1}$ \\ ${ }^{1}$ Department of Endocrinology and Metabolism, ${ }^{2}$ Department of Neurology, The Third Affiliated Hospital of Soochow University, Changzhou, \\ China \\ Contributions: (I) Conception and design: F Hua; (II) Administrative support: F Hua, XH Jiang; (III) Provision of study materials or patients: XG \\ Lian; (IV) Collection and assembly of data: C Wang, XL Huang, SK Xiang, C Yuan; (V) Data analysis and interpretation: C Wang, XL Huang; (VI) \\ Manuscript writing: All authors; (VII) Final approval of manuscript: All authors. \\ "These authors contributed equally to this work. \\ Correspondence to: Fei Hua. Department of Endocrinology and Metabolism, The Third Affiliated Hospital of Soochow University, No. 185 Juqian \\ Street, Tianning District, Changzhou, China. Email: huafei1970@suda.edu.cn.
}

\begin{abstract}
Background: The impact of an overall healthy lifestyle on early-onset stroke is still unclear. Our study thus aimed to investigate the association of overall healthy lifestyle on early-onset stroke in Chinese hospitalized stroke patients.

Methods: This retrospective study included 821 hospitalized stroke patients from the First People's Hospital of Changzhou. An overall healthy lifestyle was defined as the presence of more than 2 of the following items: healthy diet, no smoking, normal body mass index (BMI $<24 \mathrm{~kg} / \mathrm{m}^{2}$ ), engaging in moderate to high physical activity ( $\geq 3$ times/week, and $\geq 30$ minutes each time). Early-onset stroke was defined as a stroke first occurring at 50 years old or younger.

Results: Among all participants, there were 98 early-onset stroke patients and 723 late-onset stroke patients. Early-onset patients had a lower prevalence of overall healthy lifestyles than that of late-onset patients $(\mathrm{P}<0.001)$. Multivariate logistic regression revealed that an overall healthy lifestyle significantly reduced the risk of early-onset stroke. In reference to those without an overall healthy lifestyle, the multivariate-adjusted odds ratios (ORs) for early-onset stroke among participants with an overall healthy lifestyle was 0.27 [95\% confidence interval (CI): 0.07-0.98].
\end{abstract}

Conclusions: In Chinese stroke patients, a healthy lifestyle was significantly associated with early-onset stroke. Individuals who were adhering to an overall healthy lifestyle had a lower risk of early-onset stroke compared to those who were not.

Keywords: Early-onset stroke; healthy lifestyle; late-onset stroke; overall healthy lifestyle

Submitted Dec 23, 2019. Accepted for publication Jul 28, 2020.

doi: 10.21037/apm-19-656

View this article at: http://dx.doi.org/10.21037/apm-19-656

\section{Introduction}

Stroke is the second leading cause of death and the third main cause of disability in the world, accounting for 6.5 million deaths and 113 million disability-adjusted life years (DALY) (1). Although stroke is more common among older adults, the incidence rate of stroke occurring in young and middle-aged adults has gradually increased by over $40 \%$ in the past few decades (2-5). The high healthcare costs and loss of labor productivity caused by stroke and the consequent and substantial socioeconomic burden has led to early-onset stroke being considered a significant public health issue (6). Therefore, it is of great importance to identify cost-effective strategies for the prevention and early 


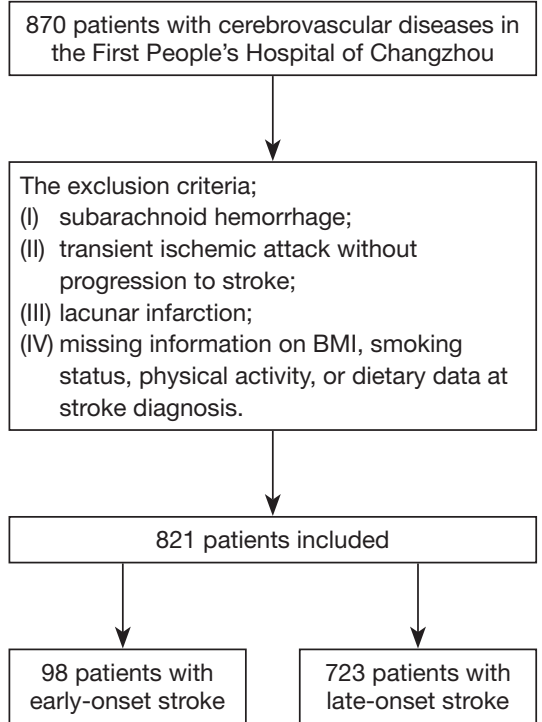

Figure 1 Flow diagram of the participant selection. BMI, body mass index.

screening of early-onset strokes.

Previous studies found that more than $90 \%$ of the stroke burden was attributable to modifiable risk factors, such as cigarette smoking, alcohol consumption, poor diet, physical inactivity, and high body mass index (BMI) $(6,7)$. Smoking, drinking, increased consumption of red meat, and high BMI were associated with an increased risk of stroke (7-9), while regular physical activity and increased consumption of fruit and vegetables significantly reduced the risk of stroke $(7,8,10)$. Moreover, adhering to an overall healthy lifestyle of not smoking, engaging in moderate to high physical activity, eating a healthy diet, and having a healthy BMI, has been associated with a substantially lower risk of stroke $(11,12)$.

However, few studies have investigated the association between a healthy lifestyle and early-onset stroke. ChoiKwon et al. reported that early-onset stroke patients were less compliant in the cessation of cigarette smoking and regular exercise than late-onset stroke patients (13). A population-based cohort study in Sweden also found the incidence rate of stroke patients who were men under the age of 45 consumed $\geq 20$ cigarettes per day, which led to them being more than 6 times more likely than nonsmoking men to have a stroke. Therefore, a dose-response association exists between smoking and risk of stroke before 45 years of age (14). Another case-control study conducted in young stroke patients revealed that an approximate $78 \%$ risk of stroke was attributable to four potentially modifiable risk factors (hypertension, low physical activity, smoking, and alcohol consumption) (15). Likewise, low green vegetable consumption was also shown to be associated with stroke occurring before the age of 50 years old (16). Nevertheless, the effect of an overall healthy lifestyle on early-onset stroke has still not been satisfactorily clarified.

Thus, in the present study, we aimed to investigate the impact of an overall healthy lifestyle on early-onset stroke among Chinese hospitalized stroke patients.

We present the following article in accordance with the STROBE reporting checklist (available at http://dx.doi. org/10.21037/apm-19-656).

\section{Methods}

\section{Study population}

This cross-sectional study was conducted over a period of 12 months (January 2017-January 2018). The study included 870 patients who were admitted to First People's Hospital of Changzhou and were diagnosed with cerebrovascular diseases. Inclusion criteria were as follows: (I) patients who agreed to participate in the study and signed the informed consent form, (II) patients underwent computed tomography (CT) or magnetic resonance imaging (MRI) scans to confirm the presence of cerebrovascular disease. The exclusion criteria included: (I) subarachnoid hemorrhage; (II) transient ischemic attack without progression to stroke; (III) lacunar infarction; (IV) missing information on BMI, smoking status, physical activity, or dietary data at stroke diagnosis. Eventually, 821 stroke patients were included (Figure 1).

This study was conducted according to the Declaration of Helsinki principles (as revised in 2013) and approved by the Institutional Review Board in the First People's Hospital of Changzhou (No. 2020025). Informed consent was obtained from all individual participants included in the study.

\section{Data collection}

Each participant completed a standard questionnaire conducted by trained interviewers for collecting sociodemographic information, medical history, and lifestyle (including smoking and drinking status, diet habit, and physical activity). Smoking status was categorized as never (including duration of passive smoking), formerly 
(including duration of quitting smoking and numbers of cigarettes when smoking), and currently (including duration and numbers of cigarettes). Drinking status was categorized as never, formerly (duration of quitting drinking), currently (including duration and alcohol consumption). Diet habit was categorized as omnivorous (balanced consumption of vegetables and meat), vegetable activists, and meat activists. The duration of habit and frequency of eating vegetables and fruits per week $(\geq 5$ or $3-4$ or $\leq 2$ days/week) were also collected. Physical activity was categorized as physically active and physically inactive. Physically active was defined as participants who participated in moderately intense physical activity $\geq 3$ times/week and $\geq 30$ minutes each time.

Anthropometric measurements were performed by trained personnel who collected information on blood pressure, height, and weight. After a 5-minute rest, blood pressure was measured twice with a 1-minute interval on the non-dominant arm (OMRON Model HEM-752 FUZZY, Omron Company, Dalian, China). The average of two measurements was used for analysis. The body weight and height of participants were measured while they were in barefoot and wearing light clothing. BMI was calculated as weight in kilograms divided by height in meters squared $\left(\mathrm{kg} / \mathrm{m}^{2}\right)$.

\section{Biochemical measurements}

Measurements were taken after at minimum of 10 hours of overnight fasting, and then venous blood samples were collected. Fasting plasma glucose (FPG) was measured using the glucose oxidase method by an autoanalyzer (AU-5800 Chemistry System, Beckman, USA). Levels of triglyceride (TG), low-density lipoprotein cholesterol (LDL-c), high-density lipoprotein cholesterol (HDL-c), and total cholesterol (TC) were measured by an autoanalyzer (AU5800 Chemistry System, Beckman, USA).

\section{Definitions}

\section{Healthy lifestyle factors}

(I) No smoking was defined as participants who had never smoked $(11,17)$. (II) Moderate to high physical activity was defined as doing moderately intense physical activity (the intensity of physical activity equal to brisk walking) $\geq 3$ times/week, and $\geq 30$ minutes each time $(18,19)$. (III) A healthy diet was defined as a balanced consumption of vegetables and meat, and consumption of vegetables at least
5 days per week, and consumption of fruit at least 5 days per week $(20,21)$. (IV) Normal BMI was defined as BMI $<24 \mathrm{~kg} / \mathrm{m}^{2}(17,20)$.

\section{Overall healthy lifestyle}

In the present study, an overall healthy lifestyle included the following healthy lifestyle factors: no smoking, moderate to high physical activity, healthy diet, and normal BMI $(11,18,22)$. Participants who met the criteria for 1 of the healthy lifestyle factors would receive 1 point; otherwise, they received 0 points. A sum of points $>2$ was defined as an overall healthy lifestyle.

\section{Early-onset stroke}

The updated definition of stroke is an acute episode of focal dysfunction of the brain, retina, or spinal cord lasting longer than $24 \mathrm{~h}$, or of any duration if imaging (CT or MRI) or autopsy showing focal infarction or hemorrhage relevant to the symptoms (23). Several editions of the International Classification of Diseases (ICD) were used to identify hemorrhagic stroke (ICD 8 and ICD 9, codes 430-431; and ICD 10, codes I60-I62), ischemic stroke (ICD 8 and ICD 9, codes 432-438; and ICD 10, codes I63-I66), and any stroke event (ICD 8 and ICD 9, codes 430-438; and ICD 10, code I60-I66) events $(22,24)$.

Early-onset stroke was defined as a stroke first occurring at 50 years old or younger. Meanwhile, late-onset stroke was defined as a stroke first occurring at 50 years old or older $(13,25)$.

\section{Statistical analyses}

In the present study, continuous variables are presented as the mean \pm standard deviation for normally distributed variables and median (interquartile) for skewed variables. Categorical variables are presented as numbers (proportions). The comparisons of characteristics between early-onset stroke patients and the other stroke patients used chi-square tests for categorical variables and Student's $t$-tests for continuous variables. Chi-square tests were used to compare the prevalence of overall healthy lifestyle and each of its component between early-onset stroke patients and late-onset stroke patients. Multivariate logistic regression was used to analyze the associations of earlyonset stroke with no smoking, moderate to high physical activity, healthy diet, normal BMI, and overall healthy lifestyle. Model 1 was adjusted for age and sex; model 2 was further adjusted for drinking status, self-reported history of 
Table 1 Characteristics of study population

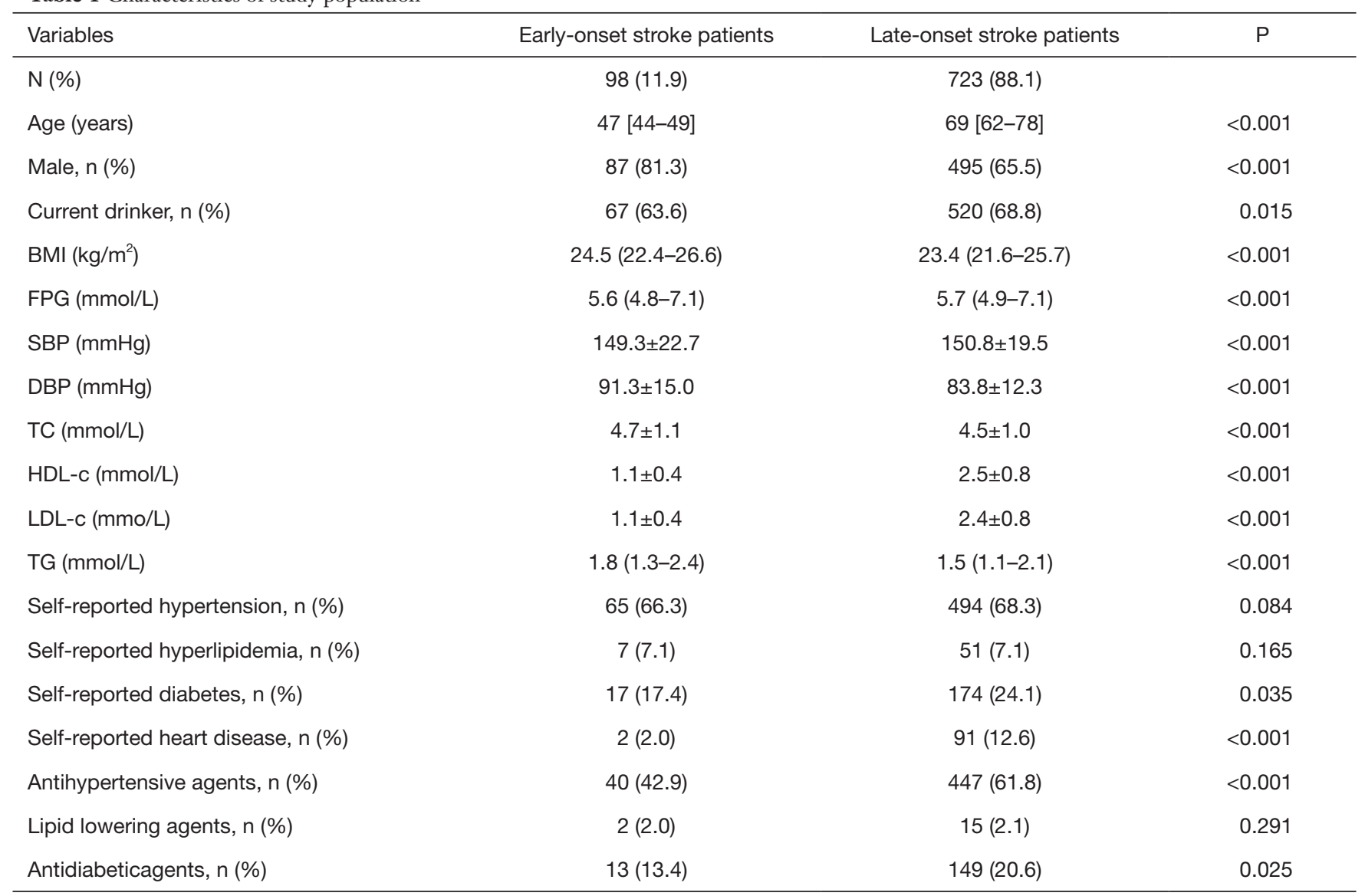

Data were presented as mean \pm SD or median (interquartile range) for continuous variables and number (\%) for categorical variables. $\mathrm{P}<0.05$ was considered statistically significant. FPG, fasting plasma glucose; DBP, diastolic blood pressure; SBP, systolic blood pressure, TC, total cholesterol; HDL-c, high-density lipoprotein cholesterol; LDL-c, low-density lipoprotein cholesterol; TG, total triglyceride.

heart disease, systolic blood pressure (SBP), and diastolic blood pressure (DBP), FPG, TG, and TC based on model 1 ; model 3 was further adjusted for antihypertensive agents, lipid-lowering agents, and antidiabetic agents based on model 2.

All analyses were conducted using the SAS software (SAS Institute, Cary, NC, USA). A two-sided $\mathrm{P}$ value $<0.05$ was considered statistically significant.

\section{Results}

\section{Characteristics of the study population}

Table 1 shows the baseline characteristics of the study population. Among all stroke patients, 98 (11.9\%) were early-onset stroke patients. Compared to late-onset stroke patients, those with early-onset stroke were younger; had more proportion of males; had higher levels of BMI, DBP, TG, and TC; but lower levels of FPG and SBP; and a lower proportion of self-reported diabetes and use of antihypertensive or antidiabetic agents (all $\mathrm{P}<0.05$ ).

\section{Prevalence of healthy lifestyle factors}

Figure 2 shows the prevalence of the overall healthy lifestyle and each of its components in the study population according to stroke-onset status. Compared to the lateonset stroke patients, the prevalence of no smoking $(\mathrm{P}=0.01)$, and healthy BMI $(\mathrm{P}<0.001)$ for early-onset stroke patients was significantly lower, but no significant difference was detected in moderate to high physical activity and healthy diet. Furthermore, the proportion of those who adhered to an overall healthy lifestyle in late-onset stroke patients was higher than that of early-onset stroke patients $(\mathrm{P}<0.001)$. 


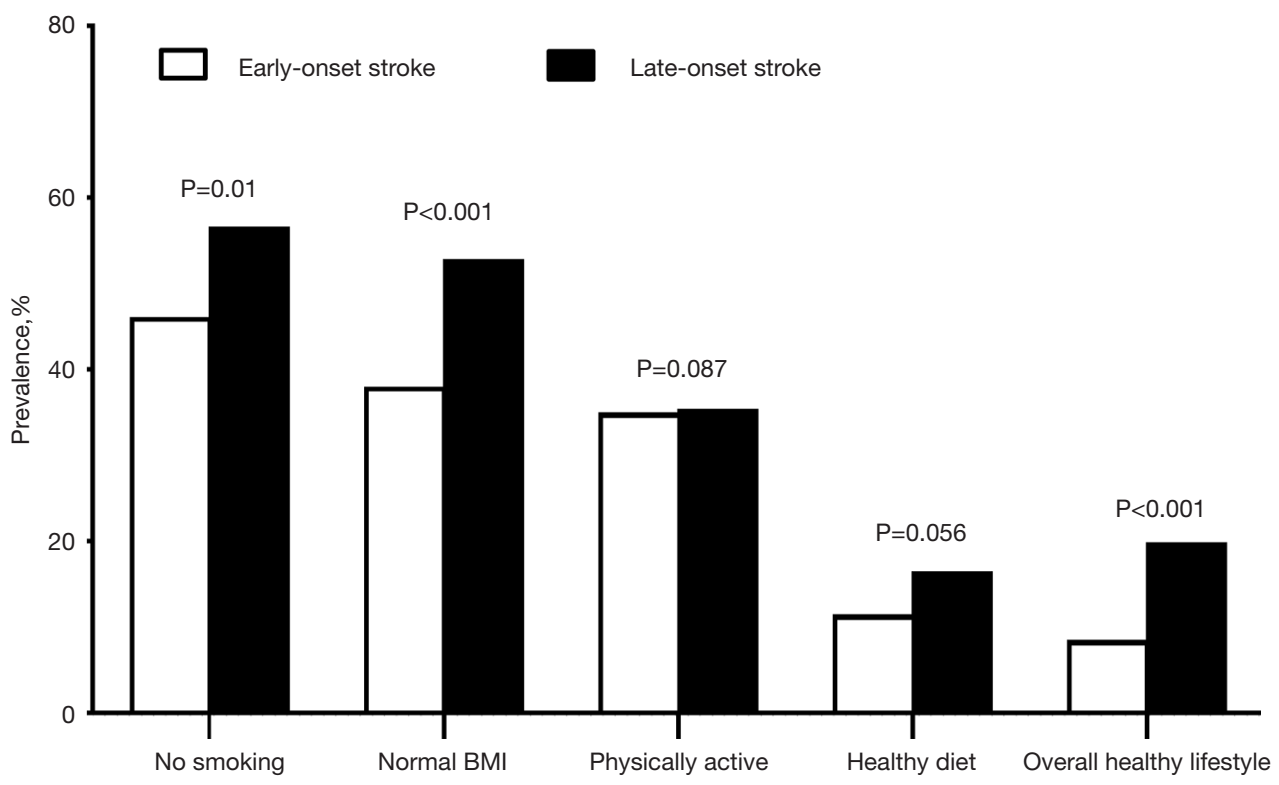

Figure 2 Prevalence of overall healthy lifestyle and each of its separate components in the study population according to stroke-onset status. Data are presented as percentages for categorical variables. A P value $<0.05$ was considered statistically significant. BMI, body mass index.

\section{Healthy lifestyle factors and the risk of early-onset stroke}

Multivariate logistic regression found that an overall healthy lifestyle was associated with a decreased risk of earlyonset stroke (Table 2). With referrence to those without an overall healthy lifestyle, the age and sex-adjusted odds ratio (OR) of early-onset stroke was $0.31(0.09-1.05)$ in participants with an overall healthy lifestyle. When further adjusted for drinking status, self-reported heart diseases, SBP, DBP, FPG, TC, and TG, the risk of early-onset stroke significantly decreased 74\% [OR: $0.26,95 \%$ confidence interval (CI): 0.07-0.95] in participants with an overall healthy lifestyle. When further adjusted for the use of antihypertensive agents, lipid-lowering agents, and glucoselowering agents, the association of an overall healthy lifestyle with early-onset stroke remained statistically significant (OR: 0.27, 95\% CI: 0.07-0.98).

\section{Discussion}

In this retrospective population-based study, we found that an overall healthy lifestyle was associated with a reduced risk of early-onset stroke. Adhering to more healthy lifestyle factors was associated with a lower risk of early-onset stroke. Our results suggest that having an overall healthy lifestyle is critical in preventing an early-onset stroke.

Stroke are a complex disease caused by both genetic and environmental factors. As genetics, age, sex, and ethnicity are non-modifiable factors, metabolic status, and lifestyle choices may be more helpful to understanding the incurrence of stroke (26). Recently, some studies have focused on the effects of lifestyle factors on early-onset stroke. A higher risk (HR: 1.88) of stroke among the current smokers versus never smokers has been observed in a population-based case-control study focusing on the risks of ischemic stroke in men aged 15 to 49 years (27). A BMI $\geq 25 \mathrm{~kg} / \mathrm{m}^{2}$ significantly increased general cardiovascular risks and specific risks of stroke including young stroke cases (28). The INTERSTROKE study, a large international casecontrol study, recruited 13,447 stroke cases and found that regular physical activity and a healthy diet reduced the risk of stroke among adults aged 55 years old or younger (OR: 0.60, 95\% CI: $0.45-0.80$ and OR: 0.68, 95\% CI: $0.55-0.86$, respectively). Current smoking and heavy alcohol intake also increased the risk of stroke (OR: 1.66, 95\% CI: 1.362.02 and OR: 2.20, 95\% CI: 1.49-3.23, respectively) (29). In our study, after adjustment for confounding factors, no statistically significant association was detected between any single lifestyle factor (no smoking, moderate to high physical activity, healthy diet, and normal BMI) and risk of early-onset stroke, which is inconsistent with previous studies. This may be attributed to the small sample size and participants recruited from a single center. However, the 
Table 2 The association of overall healthy lifestyle factors and the risk of early-onset stroke

\begin{tabular}{|c|c|c|c|}
\hline Variables & \multicolumn{3}{|c|}{ OR $(95 \% \mathrm{Cl})$} \\
\hline \multicolumn{4}{|c|}{ No smoking } \\
\hline No & 1.00 (Ref.) & 1.00 (Ref.) & 1.00 (Ref.) \\
\hline Yes & $0.96(0.38-2.32)$ & $0.87(0.35-3.28)$ & $0.95(0.37-2.46)$ \\
\hline No & 1.00 (Ref.) & 1.00 (Ref.) & 1.00 (Ref.) \\
\hline Yes & $0.75(0.35-1.58)$ & $0.67(0.30-1.46)$ & $0.67(0.30-1.48)$ \\
\hline \multicolumn{4}{|c|}{ Moderate to high physical activity } \\
\hline No & 1.00 (Ref.) & 1.00 (Ref.) & 1.00 (Ref.) \\
\hline No & 1.00 (Ref.) & 1.00 (Ref.) & 1.00 (Ref.) \\
\hline Yes & $1.40(0.41-4.78)$ & $1.40(0.39-5.06)$ & $1.46(0.40-5.27)$ \\
\hline \multicolumn{4}{|c|}{ Overall healthy lifestyle } \\
\hline No & 1.00 (Ref.) & 1.00 (Ref.) & 1.00 (Ref.) \\
\hline Yes & $0.31(0.09-1.05)$ & $0.26(0.07-0.95)$ & $0.27(0.07-0.98)$ \\
\hline
\end{tabular}

Model 1: adjusted for age (years), sex (men/women). Model 2: further adjusted for drink (yes/no), self-reported heart disease (yes/no), SBP, DBP, FPG, TG, TC based on model 1. Model 3: further adjusted for use of antihypertensive agents, lipid lowering agents and antidiabetic agents based on model 2. BMI, body mass index; OR, odds ratio; 95\% Cl, 95\% confidence interval; Ref., reference; SBP, systolic blood pressure, DBP, diastolic blood pressure; FPG, fasting plasma glucose; TG, total triglyceride; TC, total cholesterol.

overall healthy lifestyle was significantly associated with the decreased risk of early-onset stroke, which was in line with the previous studies.

The major strength of our study was the design of investigating the overall effects of healthy lifestyle factors on the risk of early-onset stroke. To our knowledge, this is the first population-based study that showed an association between an overall healthy lifestyle and a reduced risk of early-onset stroke. Additionally, the diagnosis of stroke was dependent on the imaging, which was more accurate than self-reported events in other studies. However, several limitations of our study should also be noted. Firstly, the sample size of our research was not large, with 98 earlyonset stroke cases and 723 late-onset stroke cases $(16,30)$. However, participants in the present study were measured by CT/MRI, and inpatients were diagnosed by physicians; these measures are more reliable than large sample sizes of self-reported medical histories.

Moreover, the proportion of early-onset stroke is similar to previous studies. Ekker reported that 1 in 10 strokes were in young adults (25), while in our study, 11.9\% [98] were early-onset stroke patients. Secondly, our study was retrospective in design, so a causal inference cannot be drawn, and thus the prospective study is warranted in the future. Thirdly, our study was conducted in Chinese hospitalized patients. Hence, caution should be taken in generalizing the results to other ethnicities. Finally, the information on lifestyle factors was self-reported, so some recall bias was unavoidable in our study. The additional validation of lifestyle factors should be undertaken in a future follow-up study.

\section{Conclusions}

In conclusion, the present study showed that adhering to an overall healthy lifestyle was significantly associated with a reduced risk of early-onset stroke. The power of an overall healthy lifestyle was more significant than any single component of a healthy lifestyle alone. These findings further support the current recommendation that 
patients at high risk for stroke should practice a healthy lifestyle to improve their health and maintain a lower risk of developing early-onset stroke. Further prospective studies were warranted to verify the association of an overall healthy lifestyle with the incident of early-onset stroke.

\section{Acknowledgments}

Funding: This study was supported by grants from the Special Foundations for Key Research and Development of Jiangsu Province (BE2016659), Changzhou High-Level Medical Talents Training Project (2016ZCL J020), and the Applied Basic Research Programs of Changzhou (CJ2018044).

\section{Footnote}

Reporting Checklist: The authors have completed the STROBE reporting checklist. Available at http://dx.doi. org/10.21037/apm-19-656

Data Sharing Statement: Available at http://dx.doi. org/10.21037/apm-19-656

Conflicts of Interest: All authors have completed the ICMJE uniform disclosure form (available at http://dx.doi. org/10.21037/apm-19-656). The authors have no conflicts of interest to declare.

Ethical Statement: The authors are accountable for all aspects of the work in ensuring that questions related to the accuracy or integrity of any part of the work are appropriately investigated and resolved. The present study was approved by the Institutional Review Board in the First People's Hospital of Changzhou (No. 2020025) and was conducted in compliance with the Declaration of Helsinki (as revised in 2013). Written informed consent forms were obtained from all subjects.

Open Access Statement: This is an Open Access article distributed in accordance with the Creative Commons Attribution-NonCommercial-NoDerivs 4.0 International License (CC BY-NC-ND 4.0), which permits the noncommercial replication and distribution of the article with the strict proviso that no changes or edits are made and the original work is properly cited (including links to both the formal publication through the relevant DOI and the license). See: https://creativecommons.org/licenses/by-nc-nd/4.0/.

\section{References}

1. Feigin VL, Norrving B, Mensah GA. Global burden of stroke. Circ Res 2017;120:439-48.

2. Béjot $Y$, Daubail B, Jacquin $A$, et al. Trends in the incidence of ischaemic stroke in young adults between 1985 and 2011: the Dijon Stroke Registry. J Neurol Neurosurg Psychiatry 2014;85:509-13.

3. Béjot $\mathrm{Y}$, Aouba A, de Peretti C, et al. Time trends in hospital-referred stroke and transient ischemic attack: results of a 7-year nationwide survey in France. Cerebrovasc Dis 2010;30:346-54.

4. Medin J, Nordlund A, Ekberg K, et al. Increasing stroke incidence in Sweden between 1989 and 2000 among persons aged 30 to 65 years: evidence from the Swedish Hospital Discharge Register. Stroke 2004;35:1047-51.

5. Béjot $\mathrm{Y}$, Bailly H, Durier J, et al. Epidemiology of stroke in Europe and trends for the 21st century. Presse Med 2016;45:e391-8.

6. Feigin VL, Roth GA, Naghavi M, et al. Global burden of stroke and risk factors in 188 countries, during 1990-2013: a systematic analysis for the Global Burden of Disease Study 2013. Lancet Neurol 2016;15:913-24.

7. O'Donnell MJ, Xavier D, Liu L, et al. Risk factors for ischaemic and intracerebral haemorrhagic stroke in 22 countries (the INTERSTROKE study): a case-control study. Lancet 2010;376:112-23.

8. Niewada M, Michel P. Lifestyle modification for stroke prevention: facts and fiction. Curr Opin Neurol 2016;29:9-13.

9. Liu X, Zhang D, Liu Y, et al. A J-shaped relation of $\mathrm{BMI}$ and stroke: systematic review and dose-response meta-analysis of 4.43 million participants. Nutr Metab Cardiovasc Dis 2018;28:1092-9.

10. Hu D, Huang J, Wang Y, et al. Fruits and vegetables consumption and risk of stroke: a meta-analysis of prospective cohort studies. Stroke 2014;45:1613-9.

11. Liu G, Li Y, Hu Y, et al. Influence of lifestyle on incident cardiovascular disease and mortality in patients with diabetes mellitus. J Am Coll Cardiol 2018;71:2867-76.

12. Berstad P, Botteri E, Larsen IK, et al. Lifestyle changes at middle age and mortality: a population-based prospective cohort study. J Epidemiol Community Health 2017;71:59-66.

13. Choi-Kwon S, Kwon SU, Kim JS. Compliance with risk factor modification: early-onset versus late-onset stroke patients. Eur Neurol 2005;54:204-11.

14. Falkstedt D, Wolff V, Allebeck P, et al. Cannabis, 
tobacco, alcohol use, and the risk of early stroke: a population-based cohort study of 45000 Swedish men. Stroke 2017;48:265-70.

15. Aigner A, Grittner U, Rolfs A, et al. Contribution of established stroke risk factors to the burden of stroke in young adults. Stroke 2017;48:1744-51.

16. Sarfo FS, Ovbiagele B, Gebregziabher M, et al. Stroke among young west africans: evidence from the SIREN (Stroke Investigative Research and Educational Network) large multisite case-control study. Stroke 2018;49:1116-22.

17. Lv J, Yu C, Guo Y, et al. Adherence to a healthy lifestyle and the risk of type 2 diabetes in Chinese adults. Int J Epidemiol 2017;46:1410-20.

18. Rutten-Jacobs LC, Larsson SC, Malik R, et al. Genetic risk, incident stroke, and the benefits of adhering to a healthy lifestyle: cohort study of 306473 UK Biobank participants. BMJ 2018;363:k4168.

19. Huang ZX, Lin XL, Lu HK, et al. Lifestyles correlate with stroke recurrence in Chinese inpatients with first-ever acute ischemic stroke. J Neurol 2019;266:1194-202.

20. Lv J, Yu C, Guo Y, et al. Adherence to healthy lifestyle and cardiovascular diseases in the Chinese population. J Am Coll Cardiol 2017;69:1116-25.

21. Yang YX, Wang XL, Leong PM, et al. New Chinese dietary guidelines: healthy eating patterns and foodbased dietary recommendations. Asia Pac J Clin Nutr 2018;27:908-13.

22. Zhang Y, Tuomilehto J, Jousilahti P, et al. Lifestyle factors on the risks of ischemic and hemorrhagic stroke. Arch Intern Med 2011;171:1811-8.

23. Sacco RL, Kasner SE, Broderick JP, et al. An updated

Cite this article as: Wang C, Huang XL, Xiang SK, Lian XG, Yuan C, Jiang XH, Hua F. The effect of an overall healthy lifestyle on early-onset stroke: a cross-sectional study. Ann Palliat Med 2020;9(5):2623-2630. doi: 10.21037/apm-19-656 definition of stroke for the 21 st century: a statement for healthcare professionals from the American Heart Association/American Stroke Association. Stroke 2013;44:2064-89.

24. Leppälä JM, Virtamo J, Heinonen OP. Validation of stroke diagnosis in the National Hospital Discharge Register and the Register of Causes of Death in Finland. Eur J Epidemiol 1999;15:155-60.

25. Ekker MS, Boot EM, Singhal AB, et al. Epidemiology, aetiology, and management of ischaemic stroke in young adults. Lancet Neurol 2018;17:790-801.

26. Boehme AK, Esenwa C, Elkind MS. Stroke risk factors, genetics, and prevention. Circ Res 2017;120:472-95.

27. Markidan J, Cole JW, Cronin CA, et al. Smoking and risk of ischemic stroke in young men. Stroke 2018;49:1276-8.

28. Global Burden of Metabolic Risk Factors for Chronic Diseases Collaboration (BMI Mediated Effects), Lu Y, Hajifathalian K, et al. Metabolic mediators of the effects of body-mass index, overweight, and obesity on coronary heart disease and stroke: a pooled analysis of 97 prospective cohorts with 1.8 million participants. Lancet 2014;383:970-83.

29. O'Donnell MJ, Chin SL, Rangarajan S, et al. Global and regional effects of potentially modifiable risk factors associated with acute stroke in 32 countries (INTERSTROKE): a case-control study. Lancet 2016;388:761-75.

30. von Sarnowski B, Putaala J, Grittner U, et al. Lifestyle risk factors for ischemic stroke and transient ischemic attack in young adults in the Stroke in Young Fabry Patients study. Stroke 2013;44:119-25. 